\title{
Über die Struktur und das Hochdruckverhalten des $\mathrm{B}-\mathrm{Li}_{6} \mathrm{UO}_{6}$
}

\author{
The Crystal Structure and the High Pressure Stability of $\beta-\mathrm{Li}_{6} \mathrm{UO}_{6}$
}

\section{JÜRGEN HAUCK}

Institut für Festkörperforschung, Kernforschungsanlage Jülich

und

\section{Matthias Rosenhauer}

Institut für Petrologie der Universität Frankfurt/Main

(Z. Naturforsch. 31 b, 1053-1057 [1976]; eingegangen am 7. April 1976)

Crystal Structure, Lithium-uranate(VI), IR, High Pressure Stability

\begin{abstract}
$\mathrm{Li}_{6} \mathrm{UO}_{6}$ has a reversible phase transformation at $680^{\circ} \mathrm{C}$ and decomposes above about $850{ }^{\circ} \mathrm{C}$. At high pressure the low temperature modification becomes unstable because of an invariant point in the system $\mathrm{Li}_{2} \mathrm{O}-\mathrm{Li}_{4} \mathrm{UO}_{5}$ at approximately $13 \mathrm{~Kb}$ and $620{ }^{\circ} \mathrm{C} . \beta-\mathrm{Li}_{6} \mathrm{UO}_{6}$ has a triclinic unit cell with $\alpha=5.203, b=5.520, c=5.536 \AA, \alpha=114.7, \beta=120.7$ and $\gamma=75.5^{\circ}$. The close relationship between the crystal structures of $\mathrm{Li}_{6} \mathrm{TeO}_{6}$ and $\mathrm{Li}_{6} \mathrm{UO}_{6}$ is also suggested from similar infrared spectra and from partial solid solution $\mathrm{Li}_{6} \mathrm{UO}_{6}-\mathrm{Li}_{6} \mathrm{TeO}_{6}$.
\end{abstract}

Lithiumoxid und $\mathrm{Li}_{4} \mathrm{UO}_{5}$ reagieren im Sauerstoffstrom oberhalb von $540 \pm 10^{\circ} \mathrm{C}$ zu $\alpha-\mathrm{Li}_{6} \mathrm{UO}_{6}$. Bei $680 \pm 10^{\circ} \mathrm{C}$ wandelt sich die Verbindung reversibel

Sonderdruckanforderungen an Dr. JüRGEN HAUCK, Institut für Festkörperforschung, Kernforschungsanlage Jülich, Postfach 1913, D-5170 Jülich. in eine zweite Modifikation $\beta-\mathrm{Li}_{6} \mathrm{UO}_{6}$ um, die sich im offenen System oberhalb von ca. $850^{\circ} \mathrm{C}$ zu $\mathrm{Li}_{4} \mathrm{UO}_{5}$ zersetzt, wobei $\mathrm{Li}_{2} \mathrm{O}$ wegsublimiert ${ }^{1}$. Im $\alpha-\mathrm{Li}_{6} \mathrm{UO}_{6}$ nehmen isolierte $\mathrm{UO}_{6}$-Oktaeder die Eckplätze eines rhomboedrisch verzerrten Kubus ein ${ }^{2}$ (Tab. I). Die sechs Sauerstoffatome der $\mathrm{UO}_{6}$ -

Tab. I. Gitterkonstanten und Molvolumen von verschiedenen Lithiumhexaoxometallaten sowie dem Zweikomponentengemisch $\mathrm{Li}_{2} \mathrm{O} / \mathrm{Li}_{4} \mathrm{UO}_{5}$ im Vergleich zu $\beta-\mathrm{Li}_{6} \mathrm{UO}_{6}$.

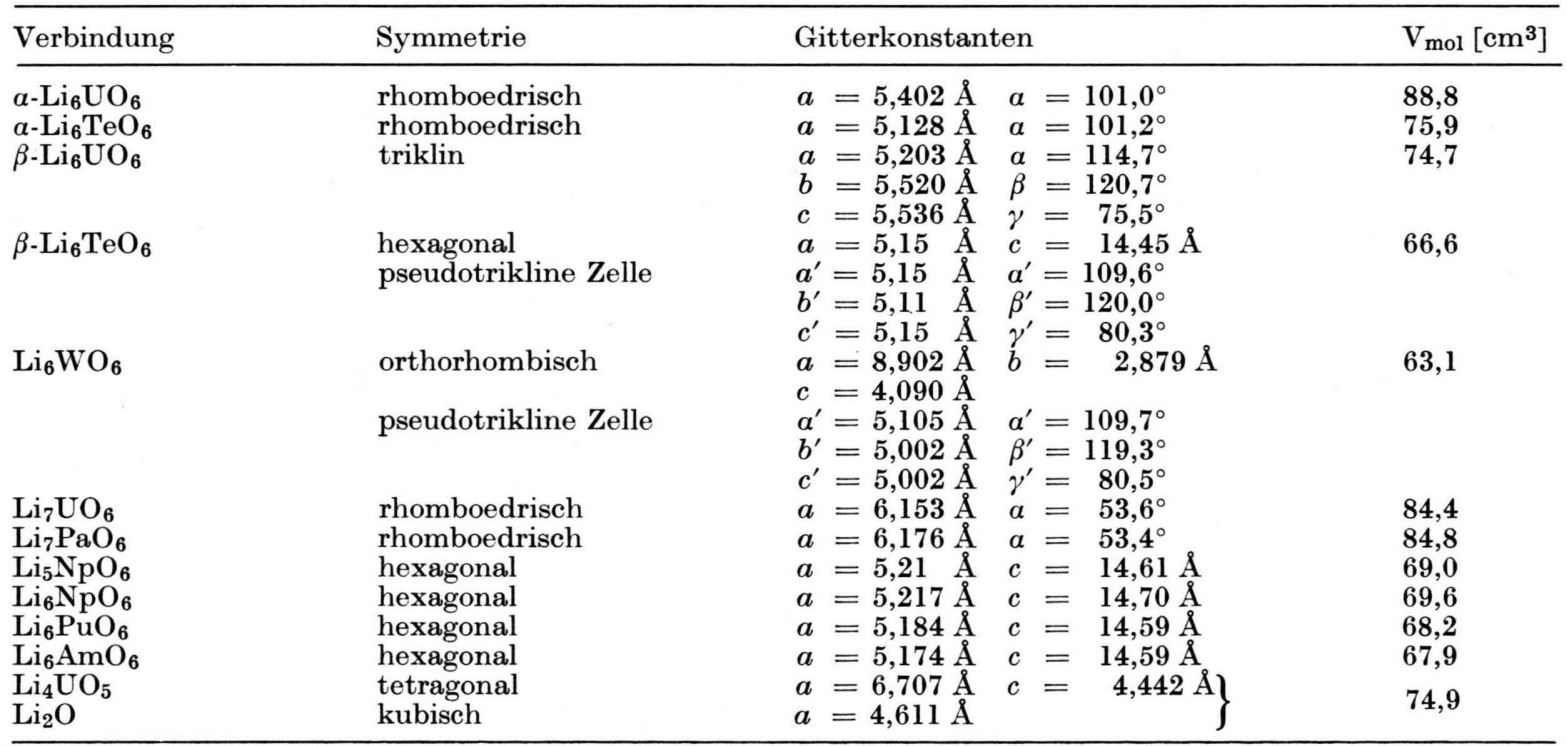


Oktaeder sind einheitlich gebunden mit einem Bindungsabstand von $2,27 \pm 5 \AA$. Die Kristallstruktur ist ähnlich wie die $\mathrm{Li}_{6} \mathrm{TeO}_{6}-\mathrm{Struktur}^{3}$, in der die Oktaeder jedoch um etwa $20^{\circ}$ um die dreizählige Achse gedreht sind. Hierbei wird aus der fünffachen Koordination der Lithiumatome in $\mathrm{Li}_{6} \mathrm{TeO}_{6}$ eine verzerrte tetraedrische Koordination im $\alpha-\mathrm{Li}_{6} \mathrm{UO}_{6}$. In beiden Strukturen ist die Packungsdichte der voluminösen Sauerstoffatome relativ gering im Vergleich zu anderen Hexaoxometallaten. Hochdruckexperimente ergaben bei $\mathrm{Li}_{6} \mathrm{TeO}_{6}$ eine zweite Modifikation oberhalb von $22 \mathrm{~kb}$ bei $700{ }^{\circ} \mathrm{C}$. Im $\beta-\mathrm{Li}_{6} \mathrm{TeO}_{6} \quad\left(\beta-\mathrm{Li}_{6} \mathrm{ReO}_{6}\right.$-Struktur $) \quad$ bilden die Sauerstoffatome annähernd eine kubisch dichteste Kugelpackung, wobei das Molvolumen um 12,2\% kleiner ist als in der $\alpha-\mathrm{Li}_{6} \mathrm{TeO}_{6}$-Struktur (Tab. I).

\section{Hochdruckexperimente im System $\mathbf{L i}_{4} \mathbf{U O}_{5}-\mathbf{L i}_{2} \mathbf{O}$}

Die Hochdruckexperimente wurden in einer ,belt"-Apparatur 5 im Temperaturbereich von $c a$. 550 bis $800^{\circ} \mathrm{C}$ durchgeführt. In vorhergehenden Versuchen ${ }^{1}$ war festgestellt worden, daß die Gleichgewichtseinstellung bei niedrigen Temperaturen zu langsam ist und daß sich $\mathrm{Li}_{6} \mathrm{UO}_{6}$ bei höheren Temperaturen zersetzt. Die Druckeichung wurde mit Hilfe der polymorphen Umwandlung Bi I-II, Bi II-III und Tl II-III sowie mit der Quarz-CoesitGleichgewichtskurve durchgeführt (zur Diskussion der Fehler der Temperatur- und Druckmessungen s. 1.c. ${ }^{5}$. Die Hochdruckexperimente ergaben für die Bildung von $\alpha-\mathrm{Li}_{6} \mathrm{UO}_{6}$ aus $\mathrm{Li}_{2} \mathrm{O}$ und $\mathrm{Li}_{4} \mathrm{UO}_{5}$ einen

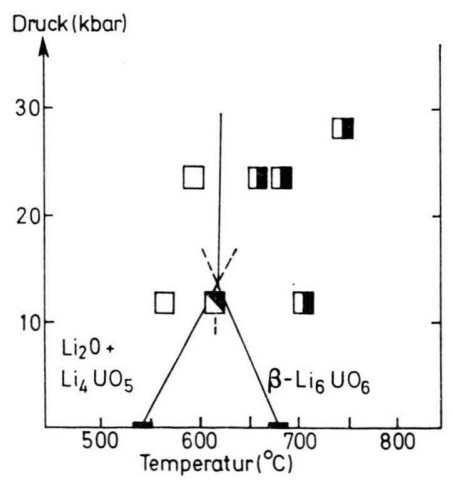

Abb. 1. p/T-Phasendiagramm für das binäre System $\mathrm{Li}_{2} \mathrm{O}-\mathrm{Li}_{4} \mathrm{UO}_{5}$. Ausgangsmaterial waren Einzelphasen oder Mischungen aus diesen. Symbole geben die Rich. tung der Reaktion an: $\square$ Zunahme von $\mathrm{Li}_{4} \mathrm{UO}_{5}$; II Zunahme von $\beta$ - $\mathrm{Li}_{6} \mathrm{UO}_{6}$; $\mathbf{v e r i n g f u ̈ g i g e ~ Z u n a h m e ~}$ von $\alpha-\mathrm{Li}_{6} \mathrm{UO}_{6}$, alle übrigen Phasen bleiben unverändert. positiven $\mathrm{dP} / \mathrm{dT}$-Wert, während für die Umwandlung von $\alpha-\mathrm{Li}_{6} \mathrm{UO}_{6}$ in $\beta-\mathrm{Li}_{6} \mathrm{UO}_{6}$ ein negativer $\mathrm{d} \mathrm{P} / \mathrm{dT}$-Wert ermittelt wurde. Oberhalb von $c a$. $13 \mathrm{~Kb}$ und $620^{\circ} \mathrm{C}$ ist $\alpha-\mathrm{Li}_{6} \mathrm{UO}_{6}$ instabil (Abb. 1), während $\beta-\mathrm{Li}_{6} \mathrm{UO}_{6}$ auch noch bei höheren Drücken existiert. Die Bildung von $\beta-\mathrm{Li}_{6} \mathrm{UO}_{6}$ aus $\mathrm{Li}_{2} \mathrm{O}$ und $\mathrm{Li}_{4} \mathrm{UO}_{5}$ ist nahezu druckunabhängig bei $c a .620^{\circ} \mathrm{C}$. Aus der Differenz der Molvolumina $4 \mathrm{~V}$ (Tab. I) und den ermittelten $(\mathrm{d} \mathrm{P} / \mathrm{dT})$-Werten läßt sich für die drei Reaktionsgleichungen die folgende Beziehung für die Entropieänderungen $\Delta \mathrm{S}$ aufstellen, die näherungsweise erfüllt wird.

(1) $\mathrm{Li}_{2} \mathrm{O}+\mathrm{Li}_{4} \mathrm{UO}_{5} \rightleftharpoons \alpha-\mathrm{Li}_{6} \mathrm{UO}_{6}$ $\Delta \mathrm{S}_{1}=(\mathrm{dP} / \mathrm{dT})_{1} \Delta \mathrm{V}_{1}$

(2) $\alpha-\mathrm{Li}_{6} \mathrm{UO}_{6} \rightleftharpoons \beta-\mathrm{Li}_{6} \mathrm{UO}_{6}$

$\Delta \mathrm{S}_{2}=(\mathrm{dP} / \mathrm{dT})_{2} \Delta \mathrm{V}_{2}$

(3) $\mathrm{Li}_{2} \mathrm{O}+\mathrm{Li}_{4} \mathrm{UO}_{5} \rightleftharpoons \beta-\mathrm{Li}_{6} \mathrm{UO}_{6}$

$\Delta \mathrm{S}_{3}=(\mathrm{dP} / \mathrm{dT})_{3} \Delta \mathrm{V}_{3}$

$\Delta \mathrm{S}_{1}+\Delta \mathrm{S}_{2}=\Delta \mathrm{S}_{3}$

Oberhalb von $c a .30 \mathrm{~Kb}$ wurde $\beta-\mathrm{Li}_{6} \mathrm{UO}_{6} \mathrm{zu}$ $\mathrm{Li}_{7} \mathrm{UO}_{6}$ mit fünfwertigem Uran reduziert, was wahrscheinlich auf die reduzierende Atmosphäre in dem die Probe umgebenden Pyrophyllit mit Graphitmantel zurückzuführen sein dürfte. Das Phasendiagramm läßt den Schluß zu, daß die Struktur des $\beta$ - $\mathrm{Li}_{6} \mathrm{UO}_{6}$ ähnlich wie die meisten Lithiumhexaoxometallate eine annähernd dichteste Sauerstoffpackung aufweist. Die ebenso bei hohem Druck stabilen Komponenten des $\mathrm{Z}$ weiphasengebietes $\mathrm{Li}_{2} \mathrm{O}$ und $\mathrm{Li}_{4} \mathrm{UO}_{5}$ haben beide Kristallstrukturen mit kubisch dichtesten Sauerstoffpackungen: $\mathrm{Li}_{2} \mathrm{O}$ kristallisiert in der Antifluoritstruktur und $\mathrm{Li}_{4} \mathrm{UO}_{5}$ hat eine verzerrte $\mathrm{NaCl}-$ Struktur mit eckenverknüpften $\mathrm{UO}_{6}$-Oktaederketten ${ }^{7,8}$. Das Molvolumen von $\mathrm{Li}_{2} \mathrm{O}$ und $\mathrm{Li}_{4} \mathrm{UO}_{5}$ ist wesentlich kleiner als das Molvolumen von $\alpha-\mathrm{Li}_{6} \mathrm{UO}_{6}$.

\section{Zur Kristallstruktur des $\mathbf{B}-\mathbf{L i}_{6} \mathbf{U}_{6}$}

$\beta-\mathrm{Li}_{6} \mathrm{UO}_{6}$ ist nicht isotyp zu den anderen bisher bekannten Hexaoxometallaten ${ }^{9}$. Das Röntgenpulverdiagramm (Tab. II) ließ sich triklin mit den in Tab. I angegebenen Gitterkonstanten indizieren. Der Vergleich mit den anderen Hexaoxometallaten zeigt, daß die Elementarzelle nur eine Formeleinheit $\mathrm{Li}_{6} \mathrm{UO}_{6}$ beinhaltet. Gegenüber der $\alpha$-Modifikation ist das Volumen um 15,9\% kleiner. Das molare Gemisch von $\mathrm{Li}_{2} \mathrm{O}$ und $\mathrm{Li}_{4} \mathrm{UO}_{5}$ hat ein etwas größeres Volumen (Tab. I).

Die Reflexintensitäten von $\beta-\mathrm{Li}_{6} \mathrm{UO}_{6}$ wurden mit 
Tab. II. Röntgenpulverdiagramm von $\beta$ - $\mathrm{Li}_{6} \mathrm{UO}_{6}\left(\mathrm{CuK}_{a}\right.$-Strahlung) bis $\vartheta_{\mathrm{Cu}}=27^{\circ}$.

\begin{tabular}{|c|c|c|c|c|c|c|c|}
\hline$h k l$ & $10^{4} / \mathrm{d}^{2}$ ber. & $10 / \mathrm{d}^{2}$ beob. & $I / I_{0}$ beob. & $h k l$ & $10^{4} / \mathrm{d}^{2}$ ber. & $10^{4} / \mathrm{d}^{2}$ beob. & $I / I_{0}$ beob. \\
\hline $\begin{array}{lll}0 & 1 & 0\end{array}$ & 398 & 399 & 72 & $\begin{array}{lll}2 & 1 & 0\end{array}$ & 2318 & & \\
\hline $\begin{array}{lll}1 & 0 & 0\end{array}$ & $501\}$ & 499 & 100 & $22 \overline{2}$ & 2338 & & \\
\hline $\begin{array}{lll}0 & 0 & 1\end{array}$ & $502\}$ & \pm 50 & & $0 \overline{2} 2$ & 2358 & 2361 & 33 \\
\hline $\begin{array}{lll} & 0 & 1\end{array}$ & 540 & 539 & 34 & $\begin{array}{lll}\overline{2} & 1 & 1\end{array}$ & 2373 & & \\
\hline $11 \overline{1}$ & $585\}$ & & 67 & $22 \overline{1}$ & $2381\}$ & & \\
\hline $\begin{array}{lll}0 & \overline{1} & 1\end{array}$ & $589\}$ & 081 & 67 & $\begin{array}{lll}\overline{2} & 1 & 0\end{array}$ & $2487\}$ & 2515 & 8 \\
\hline $\begin{array}{lll}1 & 1 & 0\end{array}$ & 857 & 860 & 17 & $\underline{1} \overline{2} 1$ & $2522\}$ & 2515 & 0 \\
\hline $\begin{array}{lll}\overline{1} & 1 & 0\end{array}$ & 942 & 941 & 30 & $\begin{array}{lll}\overline{1} & 1 & 2\end{array}$ & 2647 & 2650 & 5 \\
\hline $\begin{array}{llll}0 & 1 & 1\end{array}$ & 1212 & 1215 & 15 & $\begin{array}{lll}0 & 2 & 1\end{array}$ & 2719 & 2717 & 5 \\
\hline $\begin{array}{llllllllll}\overline{1} & 1 & 1\end{array}$ & 1292 & 1291 & 10 & $\begin{array}{lll}\overline{1} & 2 & 1\end{array}$ & 2841 & 2843 & 4 \\
\hline $11 \overline{2}$ & 1317 & 1315 & 13 & $\begin{array}{lll}0 & 12\end{array}$ & $3031)$ & & \\
\hline $12 \overline{1}$ & $1426)$ & & & $11 \overline{3}$ & 3054 & 3056 & 8 \\
\hline $\begin{array}{lll}1 & 0 & 1\end{array}$ & $1467\}$ & 1468 & 20 & $13 \mathrm{I}$ & 3065 & & \\
\hline $\begin{array}{lll}0 & \overline{2} & 1\end{array}$ & 1473 & & & $\begin{array}{ll}21 & 1\end{array}$ & $3123\}$ & & \\
\hline $\begin{array}{lll}\overline{2} & 0 & 1\end{array}$ & 1579 & & & $0 \overline{3} 1$ & 3154 & 3159 & 9 \\
\hline$\overline{2} 1 \overline{1}$ & 1582 & & & $13 \overline{2}$ & 3174 & & \\
\hline $\begin{array}{ccc}\overline{1} & 0 & 2\end{array}$ & 1583 & 1581 & 28 & $1 \overline{1} 2$ & $3256\}$ & & \\
\hline $\begin{array}{ll}02 & 0\end{array}$ & 1594 & & & 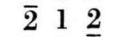 & 3265 & & \\
\hline $1 \bar{I} 1$ & 1596 & & & $12 \overline{3}$ & 3272 & 3282 & 10 \\
\hline $\begin{array}{lll}0 & \overline{1} & 2\end{array}$ & 1785 & 1790 & 5 & $2 \overline{2} \overline{3}$ & 3300 & & \\
\hline $12 \overline{2}$ & 1847\} & 1846 & 9 & $\begin{array}{lll}3 & 1 & \overline{2}\end{array}$ & 3385 & 3381 & 8 \\
\hline $2 \overline{1}$ & $1850\}$ & 1040 & 9 & $\begin{array}{lll}2 & 2 & 0\end{array}$ & $3429]$ & & \\
\hline $\begin{array}{lll}2 & 0 & 0\end{array}$ & 2004 & & & $\begin{array}{lll}2 & 0 & 1\end{array}$ & 3434 & 3432 & 11 \\
\hline $\begin{array}{lll}0 & 0 & 2\end{array}$ & $2010\}$ & 2009 & 24 & $\begin{array}{lll}102 & 2\end{array}$ & 3438 & & \\
\hline 120 & 2010 J & & & & & & \\
\hline $\begin{array}{lll}1 & 1 & 1\end{array}$ & 2135 & & & & & & \\
\hline $\begin{array}{lll}\overline{2} & 0 & 2\end{array}$ & $2159\}$ & 2154 & 36 & & & & \\
\hline$\overline{1} 20$ & $2179]$ & & & & & & \\
\hline
\end{tabular}

einem Pulverdiffraktometer mit Schrittschaltwerk gemessen, wobei 15 nicht überlagerte und 17 überlagerte Reflexe für die Strukturfaktorrechnung zur Bestimmung der Atomlagen erhalten wurden. Eine Strukturfaktorrechnung allein unter Berücksichtigung der schweren Uranatome auf 000 erbrachte bereits einen $R$-Wert von 0,09 für die nicht überlagerten Reflexe. Für die Sauerstoffatome und Lithiumatome kommen jeweils 6 einzählige Lagen $x y z$ der Raumgruppe $\mathrm{P} 1$ oder jeweils 3 zweizählige Lagen $x y z$ der Raumgruppe $\mathrm{P} \overline{\mathbf{l}}$ in Betracht. Eine weitergehende Strukturbestimmung war wegen der Vielzahl der freien Parameter aus den Intensitätswerten des Pulverdiagramms allein nicht möglich. Aus einem Vergleich mit den Strukturen anderer Lithiumhexaoxometallate kann jedoch ein Strukturvorschlag als wahrscheinlich angenommen werden. Bei den 35 bisher untersuchten Lithiumhexaoxometallaten $^{9}$ mit Ausnahme von $\alpha-\mathrm{Li}_{6} \mathrm{UO}_{6}$ und $\alpha-\mathrm{Li}_{6} \mathrm{TeO}_{6}$ bilden die relativ voluminösen Sauerstoffatome annähernd dichteste Kugelpackungen. Es lag daher die Vermutung nahe, die auch durch die Hochdruckversuche bestärkt wurde, daß auch im $\beta-\mathrm{Li}_{6} \mathrm{UO}_{6}$ eine annähernd dichteste Sauerstoffpackung vorliegt. Bei Lithiumhexaoxometallaten mit hohem Lithiumgehalt $\left(\mathrm{Li}_{8} \mathrm{SnO}_{6}-\right.$ und $\mathrm{Li}_{7} \mathrm{SbO}_{6}$ Struktur) wurden rhomboedrische Strukturen mit annähernd hexagonal dichtesten Sauerstoffpackungen gefunden. Lithiumoxometallate mit kleinerem Lithiumgehalt $\left(\mathrm{Li}_{5} \mathrm{ReO}_{6-}, \beta-\mathrm{Li}_{6} \mathrm{ReO}_{6}\right.$ - und $\mathrm{Li}_{6} \mathrm{WO}_{6}-$ Struktur) haben dagegen eine annähernd kubisch dichteste Sauerstoffpackung mit hexagonaler oder orthorhombischer Elementarzelle. Bei einem Vergleich der rhomboedrischen bzw. pseudotriklinen Zellen mit einer Formeleinheit Lithiumhexaoxometallat (Tab. I) liegt die Vermutung nahe, daß $\beta$ - $\mathrm{Li}_{6} \mathrm{UO}_{6}$ ebenso wie $\beta-\mathrm{Li}_{6} \mathrm{TeO}_{6}$ und $\mathrm{Li}_{6} \mathrm{WO}_{6}$ eine annähernd kubisch dichteste Sauerstoffpackung bildet. Die Sauerstoffatome sollten hierbei näherungsweise folgende Atomlagen der Raumgruppe $\mathrm{P} \overline{1}$ einnehmen.

$\begin{array}{llll} & x & y & z \\ \mathrm{O}_{\mathrm{I}} & 0 & 1 / 4 & 3 / 4 \\ \mathrm{O}_{\mathrm{II}} & 1 / 3 & 1 / 4 & 5 / 12 \\ \mathrm{O}_{\mathrm{III}} & 2 / 3 & 1 / 4 & 1 / 12\end{array}$

Die Lithiumatome sind wahrscheinlich in den Tetraeder- und Oktaederlücken der Sauerstoffpackung angeordnet. Ähnlich wie $\mathrm{Li}_{6} \mathrm{WO}_{6}$ und $\beta$ - $\mathrm{Li}_{6} \mathrm{TeO}_{6}$ kann auch die $\beta-\mathrm{Li}_{6} \mathrm{UO}_{6}$-Struktur als Überstruktur des NaCl-Gitters dargestellt werden 


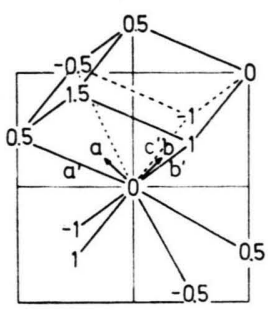

$\mathrm{Li}_{6} \mathrm{WO}_{6}$

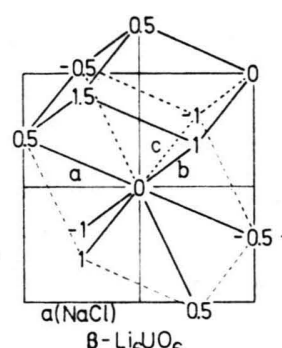

$\beta-\mathrm{Li}_{6} \mathrm{UO}_{6}$

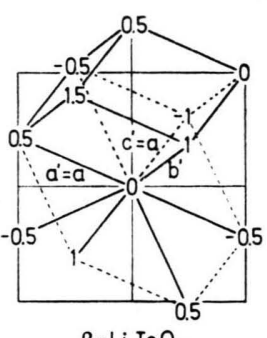

$\beta-\mathrm{Li}_{6} \mathrm{TeO}_{6}$
Abb. 2. Lage der 8 nächsten $\mathrm{XO}_{6}$-Oktaeder in $\mathrm{Li}_{6} \mathrm{WO}_{6}$, $\beta-\mathrm{Li}_{6} \mathrm{UO}_{6}$ und $\beta$ - $\mathrm{Li}_{6} \mathrm{TeO}_{6}$ mit den Translationen $a a / 2 a / 2$ der $\mathrm{NaCl}$-Überstrukturen.

(Abb. 2). Durch die Translation $a a / 2 a / 2$ ( $a=$ Gitterkonstante des $\mathrm{NaCl}$-Gitters) werden jeweils 8 nächste $\mathrm{XO}_{6}$-Oktaeder erreicht. Im $\beta$ - $\mathrm{Li}_{6} \mathrm{TeO}_{6}$ und $\beta$ - $\mathrm{Li}_{6} \mathrm{UO}_{6}$ liegen hiervon $6 \mathrm{X}$-Atome in einer hexagonalen bzw. pseudohexagonalen Schicht und die Strukturen unterscheiden sich nur hinsichtlich der Anordnung benachbarter Schichten.

Ein Vergleich der IR-Spektren (Tab. III) zeigt

Tab.III. IR-Spektren von $\alpha, \beta-\mathrm{Li}_{6} \mathrm{TeO}_{6}$ und $\alpha, \beta-\mathrm{Li}_{6} \mathrm{UO}_{6}$ im Bereich von $300-1000 \mathrm{~cm}^{-1}$

(KBr-Tabletten-Technik).

\begin{tabular}{lllll}
\hline & $v_{3}\left(\mathrm{~F}_{1 \mathrm{u}}\right)$ & $\begin{array}{c}v(\mathrm{LiO}) \\
{\left[\mathrm{cm}^{-1}\right]}\end{array}$ & $v_{4}\left(\mathrm{~F}_{1 \mathrm{u}}\right)$ & $\mathrm{f}[\mathrm{mdyn} / \AA]$ \\
\hline$\alpha-\mathrm{Li}_{6} \mathrm{TeO}_{6}$ & 640 & \multicolumn{2}{c}{470} & 3,16 \\
$\alpha-\mathrm{Li}_{6} \mathrm{UO}_{6}$ & 570 & \multicolumn{4}{c}{470} & \\
$\beta-\mathrm{Li}_{6} \mathrm{TeO}_{6}$ & 685 & 470 & 420 & 3,63 \\
$\beta-\mathrm{Li}_{6} \mathrm{UO}_{6}$ & 675,630 & 470 & 415 & 3,$69 ; 3,21$ \\
\hline
\end{tabular}

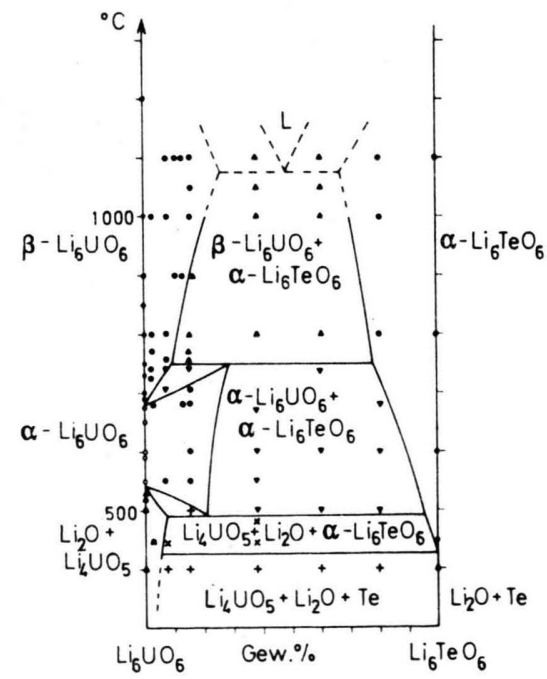

Abb. 3. Phasenbeziehungen im System $\mathrm{Li}_{6} \mathrm{UO}_{6}-\mathrm{Li}_{6} \mathrm{TeO}_{6}$ und $\mathrm{Li}_{6} \mathrm{UO}_{6}-\mathrm{Li}_{6} \mathrm{WO}_{6}$. ebenso die Ähnlichkeit zwischen $\alpha-\mathrm{Li}_{6} \mathrm{UO}_{6}$ und $\alpha-\mathrm{Li}_{6} \mathrm{TeO}_{6}$ sowie zwischen $\beta-\mathrm{Li}_{6} \mathrm{UO}_{6}$ und $\beta-\mathrm{Li}_{6} \mathrm{TeO}_{6}$. Die LiO-Schwingungen liegen jeweils bei $470 \mathrm{~cm}^{-1}$ und sind bei beiden $\alpha$-Modifikationen den $v_{4}\left(\mathrm{~F}_{1 \mathrm{u}}\right)$ Schwingungen überlagert. In den $\beta$-Modifikationen liegen die $v_{4}\left(\mathrm{~F}_{1 \mathrm{u}}\right)$-Schwingungen bei 420 bzw. $415 \mathrm{~cm}^{-1}$. Deutlich unterscheiden sich jedoch die $v_{3}\left(\mathbf{F}_{1 \mathrm{u}}\right)$-Schwingungen aufgrund der unterschiedlichen Massen von Te und U sowie der unterschiedlichen Bindungsstärke. Im IR-Spektrum von $\beta-\mathrm{Li}_{6} \mathrm{UO}_{6}$ ist neben einer starken Bande bei $630 \mathrm{~cm}^{-1}$ noch eine schwächere Bande bei $675 \mathrm{~cm}^{-1}$, die auf eine schwache Uranylbindung hinweist. Da die $\beta$ - $\mathrm{Li}_{6} \mathrm{UO}_{6}$-Struktur mit keinem anderen Hexaoxometallat isotyp ist, kann vermutet werden, daß die gegenüber $\beta-\mathrm{Li}_{6} \mathrm{TeO}_{6}$ trikline Verzerrung auf die Uranylbindungen zurückzuführen ist. Bei starken Uran-Sauerstoff bindungen werden fast immer die Sauerstoffatome unterschiedlich stark gebunden. $\alpha-\mathrm{Li}_{6} \mathrm{UO}_{6}$ ist eine der wenigen Verbindungen, in der die oktaedrisch koordinierten Sauerstoffatome gleichmäßig mit schwachen U-O-Bindungen gebunden werden. Die nach einem modifizierten Zweimassenmodell 1o berechneten Kraftkonstanten zeigen, da $\beta$ die $\mathrm{U}-\mathrm{O}$-Bindungen in $\beta-\mathrm{Li}_{6} \mathrm{UO}_{6}$ wesentlich stärker sind als in $\alpha-\mathrm{Li}_{6} \mathrm{UO}_{6}$. Die dem Uran benachbarten sechswertigen Actinid-Elemente $\mathrm{Np}$, $\mathrm{Pu}$ und $\mathrm{Am}$ bilden Verbindungen mit $\beta-\mathrm{Li}_{6} \mathrm{ReO}_{6}$ Struktur ${ }^{9,11}$, wobei das Molvolumen infolge der Actinidenkontraktion abnimmt. Auch in der Reihe $\mathrm{Li}_{7} \mathrm{PaO}_{6}, \quad \beta-\mathrm{Li}_{6} \mathrm{UO}_{6}, \quad \mathrm{Li}_{5} \mathrm{NpO}_{6}{ }^{12}$ lassen sich die Volumina sinnvoll vergleichen (Tab. I).

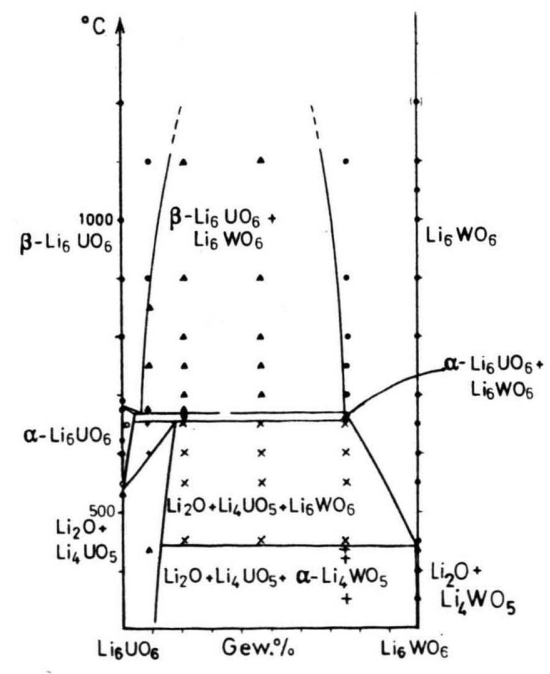




\section{Phasenumwandlungen im System}

$\mathbf{L i}_{6} \mathrm{UO}_{6}-\mathbf{L i}_{6} \mathbf{T e O}_{6}$ und $\mathbf{L i}_{6} \mathrm{UO}_{6}-\mathbf{L i}_{6} \mathrm{WO}_{6}$

Einen weiteren Hinweis über die strukturelle Verwandtschaft der verschiedenen $\mathrm{Li}_{6} \mathrm{XO}_{6}$-Strukturen erhält man auch aus einem Vergleich der pseudobinären Phasendiagramme $\mathrm{Li}_{6} \mathrm{UO}_{6}-\mathrm{Li}_{6} \mathrm{WO}_{6}$ und $\mathrm{Li}_{6} \mathrm{UO}_{6}-\mathrm{Li}_{6} \mathrm{TeO}_{6}$ (Abb. 3). Präparationstechnik und Analysenmethoden für das System $\mathrm{Li}_{6} \mathrm{UO}_{6}$ $\mathrm{Li}_{6} \mathrm{TeO}_{6}$ waren ähnlich wie für das System $\mathrm{Li}_{6} \mathrm{UO}_{6}-$ $\mathrm{Li}_{6} \mathrm{WO}_{6}{ }^{1}$. Wegen der Zersetzung von $\mathrm{Li}_{6} \mathrm{UO}_{6}$ bei höheren Temperaturen unter Verflüchtigung von $\mathrm{Li}_{2} \mathrm{O}$ konnte das System hier nur unvollständig unter-

1 J. HaUCK, J. Inorg. Nucl. Chem. 36, 2291 [1974].

2 J. HaUCK, Z. Naturforsch. 28b, 215 [1973].

3 J. Hauck, Z. Naturforsch. 25 b, 749 [1970] bzw. 24 b, 647 [1969].

4 J. Hauck und A. Hirschberg, Z. Naturforsch. 24 b, 1656 [1969].

5 A. Hirschberg, M. Rosenhauer, A. Lamprecht und K. v. Gehlen, Neues Jb. für Mineralogie, Mh. $1969,454$.

6 J. HAUCK, Z. Naturforsch. 24b, 251 [1969]. sucht werden. Die enge strukturelle Beziehung zwischen $\alpha-\mathrm{Li}_{6} \mathrm{UO}_{6}$ und $\alpha-\mathrm{Li}_{6} \mathrm{TeO}_{6}$ zeigt sich insbesondere in dem relativ großen Stabilitätsbereich der $a-\mathrm{Li}_{6} \mathrm{UO}_{6}$-Struktur mit $\mathrm{Li}_{6} \mathrm{TeO}_{6}$ als Mischkristall im Vergleich zu dem kleineren Bereich im System $\mathrm{Li}_{6} \mathrm{UO}_{6}-\mathrm{Li}_{6} \mathrm{WO}_{6} . \mathrm{Li}_{6} \mathrm{WO}_{6}, \mathrm{Li}_{6} \mathrm{UO}_{6}$ und $\mathrm{Li}_{6} \mathrm{TeO}_{6}$ sind jeweils nur bei höheren Temperaturen stabil und zersetzen sich bei $440{ }^{\circ} \mathrm{C}\left(\mathrm{Li}_{6} \mathrm{WO}_{6}\right), 540{ }^{\circ} \mathrm{C}$ $\left(\mathrm{Li}_{6} \mathrm{UO}_{6}\right)$ und $425^{\circ} \mathrm{C}\left(\mathrm{Li}_{6} \mathrm{TeO}_{6}\right)$ zu den basenärmeren Verbindungen. $\mathrm{Li}_{6} \mathrm{TeO}_{6}$ zerfällt wahrscheinlich direkt $\mathrm{zu}$ elementarem Tellur und $\mathrm{Li}_{2} \mathrm{O}$ bei $1 \mathrm{~atm}$ Sauerstoffpartialdruck.

7 L. M. Kovba, J. Struct. Chem. 3, 146 [1962].

8 M. Hoekstra und S. Siegel, J. Inorg. Nucl. Chem. 26, 693 [1964].

9 J. HAUCK, Z. Naturforsch. 24b, 1067 [1969].

$10 \mathrm{~J}$. Hauck und A. Fadini, Z. Naturforsch. 25 b, 422 [1970].

11 C. Keller, K. H. Walter und L. КосH, J. Inorg. Nucl. Chem. 27, 1205 [1965].

12 C. Keller und H. Seiffert, Inorg. Nucl. Chem. Lett. 5, 51 [1969]. 